\title{
Performance Evaluation of Energy Efficient Distance Based LEACH Protocol for Wireless Sensor Networks
}

\author{
Malothu Amru, R P Singh, S Pothalaiah
}

\begin{abstract}
The zone of wireless sensor networks (WSNs) is one of the developing and quickly developing fields in the logical world. This has realized growing ease, low-control and multiwork sensor hubs. Nonetheless, the real certainty that sensor hubs come up short on vitality rapidly has been an issue and numerous vitality effective directing conventions have been proposed to take care of this issue and safeguard the life span of the network. The proposed EEDBLEACH chooses bunch head with most elevated leftover vitality in every communication round of transmission and furthermore considers, the briefest good ways from the base station to the hubs in the group. By accepting the separation as a parameter which diminishes the vitality utilization. The EEDBLEACH Protocol is correlation with the current LEACH convention. Reproduction results demonstrate that EEDBLEACH Protocol drags out the lifetime of the network
\end{abstract}

Key Words: Wireless Sensor Networks, EEDBLEACH, LEACH.

\section{INTRODUCTION}

In the vitality proficient routing protocols clustering is done consistently and select group head $(\mathrm{CH})$ in light of residual vitality and separation of the hubs. This convention is changed adaptation of LEACH convention. The race of Cluster Head $(\mathrm{CH})$ in EEDBLEACH convention depends on outstanding vitality and the separation between other hubs and sink hub. The vitality utilization of sensor hubs depends on the separation gone by bundle, if hub transmits longer separation it expends more vitality than shorter separation. In this convention separation between hubs is processed utilizing separation condition equation. In this convention for group head race acquire normal separation among hubs and the hub chose as bunch head as for uniform good ways from every single other hub.

In the wireless sensor networks the heterogeneous is a network model where the sensor hubs have divergent introductory vitality. In heterogeneous networks the quantity of bunches is processed. Let consider $m$ is a portion of the hubs in the network and ' $n$ ' is absolute number of hubs in the networks, where the ' $m$ ' bit of sensor hubs having a ' $\alpha$ ' times extra vitality than the others hubs in wireless sensor networks. These higher vitality hubs are considered as cutting edge hubs and remaining hubs of the wireless sensor network (n-m) are consider as ordinary hubs in the network. The sensor hubs are conveyed subjectively in region.

Revised Manuscript Received on September 10, 2019.

Malothu Amru, CMR Engineering College, Hyderabad, Telangana, India.

(Email: malothuamru@gmail.com)

Dr. R P Singh, Sri Satya Sai University of Technology \& Medical Science, Bhopal, M.P, India.

Dr. S Pothalaiah, Vignana Bharathi Institute of Technology, Hyderabad, Telangana, India.

(Email: pothalaiahs@gmail.com)

The bunches are orchestrated progressively in the wireless sensor network. In a group each hub progresses toward becoming $\mathrm{CH}$ (bunch head).Cluster head is chosen with likelihood of $\mathrm{P}_{-}$opt. The hubs ought to turn into a group head all things considered $\mathrm{n} \times \mathrm{P}$ _opt per round. The hubs are chosen to be group heads in the current round never again become bunch heads in the current round. The non group head hubs have a place with set $\mathrm{G}$ and so as to keep up number of bunch heads per round, the likelihood of hubs $\mathrm{G}$ to turn into a group head increments after the some round. The choice is made toward the start of each round by every hub $\mathrm{G}$ autonomously picking an arbitrary number. In the event that the irregular number is not exactly the limit $\mathrm{T}(\mathrm{s})$ then the hub turns into a group head in the current round. The edge $T(S)$ is given underneath condition 3.1

Threshold $\mathrm{T}(\mathrm{s})=$

$\left\{\begin{array}{cl}\frac{\mathrm{p}_{\text {opt }}}{1-\mathrm{p}_{\text {opt. }}\left[\mathrm{r} \cdot \mathrm{mod} .\left(\frac{1}{\mathrm{p}_{\text {opt }}}\right)\right]}, & \text { if } S \in \mathrm{G} \\ 0 & \text { otherwise }\end{array}\right.$

The determination likelihood of every hub ' $G$ ' to progress toward becoming bunch heads increments in each round and $\mathrm{G}$ is set equivalent to 1 in the last round. Round is characterizes as a timeframe where every one of the hubs in a group need to transmit their bunch head once. In this summary how the bunch head choice procedure must be adjusted accurately to manage heterogeneous model of the nodes.

\section{ENERGY DISSIPATION OF RADIO MODEL}

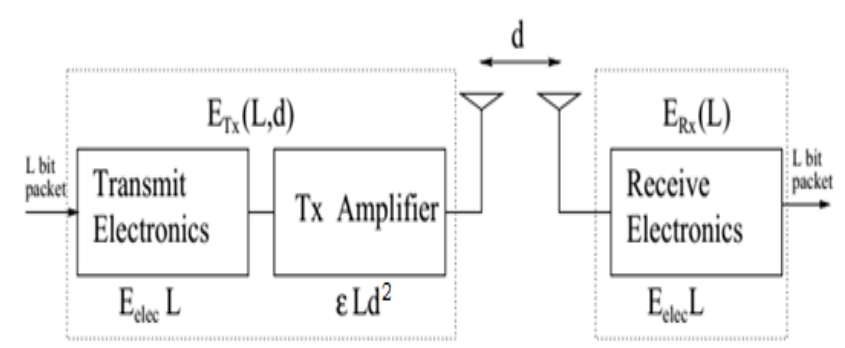

Fig. 1. Energy Dissipation of Radio Model

The consumption of energy for L bit data sending with a distance of $\mathrm{d}$ is given equation 1.2

$$
E_{T x}(l, d)= \begin{cases}\text { L. } E_{E l e c}+\text { L. } \epsilon_{\mathrm{fs}} \cdot d^{2} & , \text { if } d<d_{0} \\ \text { L. } E_{\text {Elec }}+\text { L. } \epsilon_{\mathrm{mp}} \cdot d^{4} & \text {, if } d \geq d_{0}\end{cases}
$$


In condition 1.2 E_Elec gives the disseminated vitality for each piece to run collector or transmitter circuits. $\epsilon$ ms And $\epsilon \_$fs rely upon the transmitter speaker model and $d$ is the separation between the sender and the beneficiary, by comparing the over two conditions at $\mathrm{d}=d_{o}$.

Where $\mathrm{d}_{\mathrm{o}}=\sqrt{\frac{\epsilon_{\mathrm{fs}}}{\epsilon_{\mathrm{ms}}}}$

The energy require to receive for $\mathrm{L}$ bit message is $\mathrm{E}_{\mathrm{Rx}}=$ L. $\mathrm{E}_{\mathrm{Elec}}$

Let the sensor field zone is $\mathrm{A}=\mathrm{MxM}$ square meters, in which the quantity of hubs $\mathrm{n}$ are homogeneously circulated over sensor field. The sink hub is set at center of the region (A), the separation between hubs to the sink hub or its bunch head is $\leq \mathrm{d} \_$. Condition 1.4 gives the vitality utilization of the each bunch head in the current round for accepting $\mathrm{k}$ bit information with the separation of dis

$$
E_{c h}=\frac{n}{k} L \cdot E_{D A}+\left(\frac{n}{k}-1\right) L \cdot E_{\text {Eelec }}+L \cdot E_{E l e}+L \cdot \epsilon_{f s} d_{t o B S}^{2}
$$

Where E_DA is vitality required to transmit the amassed information report to the sink hub or base station (BS). The quantity of bunches in the wireless sensor network is $\mathrm{K}$, and the normal separation between the base station (BS) and group head is $\llbracket \mathrm{d} \rrbracket$ _ to BS. The vitality dispersal for non group head hub is given by condition 1.5

$$
E_{\text {nonCH }}=L . E_{\text {ele }}+\text { L. } \epsilon_{f s} d_{\text {toCH }}^{2}(1.5)
$$

Here $d_{t o C H}$ is average distance from cluster head to its cluster member. Assume that the all the sensor nodes are uniformly distributed, as given equation 1.6

$$
d_{\text {toCH }}^{2}=\int_{x=0}^{x=x_{\max }} \int_{y=0}^{y=y_{\max }}\left(x^{2}+y^{2}\right) \rho(x, y) d x d y=\frac{M^{2}}{2 \pi k}
$$

From the above equation $1.6 \rho(x, y)$ is allocation of the nodes in the area of sensor networks. In each cluster the energy dissipated per round is equation 1.7

$$
E_{\text {cluster }}=\frac{n}{k} E_{\text {nonCH}}+E_{C H}
$$

In the network the total energy is dissipated given by the equation 1.8

(1.8)

$$
E_{\text {tot }}=L\left(n E_{D A}+2 n E_{\text {elec }}+\epsilon_{f s}\left(k d_{t o B S}^{2}+\left(n d_{\text {toCH }}^{2}\right)\right)\right.
$$

By differentiating of $E_{\text {tot }}$ W. r. t. k, this is equating to zero, the clusters optimization is

$$
k_{\text {opt }}=\sqrt{\frac{n}{2 \pi}} \frac{M}{d_{t o B S}}=\sqrt{\frac{n}{2 \pi}} \frac{2}{0.765}(1.9)
$$

The node optimal probability is calculated or selection of cluster head

$$
\mathrm{p}_{\mathrm{opt}}=\frac{\mathrm{k}_{\mathrm{opt}}}{\mathrm{n}}(1.10)
$$

The average of the distance from base station to $\mathrm{C}$ His given equation 1.11

$$
\begin{array}{r}
d_{t o B S}=\int \sqrt{x^{2}+y^{2}} \frac{1}{A} d A \\
d_{\text {toBS }}=0.765 \frac{M}{2}
\end{array}
$$

The vitality effective bunch heads are chosen in each round and in wireless sensor network burden has turned out to be all around adjusted and furthermore disseminated among every one of the hubs. The Popt is hubs ideal likelihood thinks about that hub must be to turn into a bunch head $(\mathrm{CH})$ in each round [6]. To settle on a choice whether a hub to turn into a bunch head or not the limit $\mathrm{T}(\mathrm{s})$ is given condition 1.12 .

$$
\mathrm{T}(\mathrm{s})=\left\{\begin{aligned}
\frac{\mathrm{p}_{\text {opt }}}{1-\mathrm{p}_{\text {opt. }}\left[\text { r.mod. }\left(\frac{1}{\mathrm{p}_{\text {opt }}}\right)\right]}, & \text { if } \mathrm{s} \in \mathrm{G}^{\prime} \\
0, & \text { otherwise }
\end{aligned}\right.
$$

Where Gis the arrangement of sensor hubs that have not progressed toward becoming group head inside the last $1 /$ popt round and $r$ is the current round. Toward the start of each round, every hub which has a place with the set $G$ chooses an irregular number 0 or 1 . In the event that the arbitrary number is not exactly the limit $\mathrm{T}(\mathrm{s})$ then the hub progresses toward becoming group head in the current round.

\section{ENERGY EFFICIENT DISTANCE BASED LEACH}

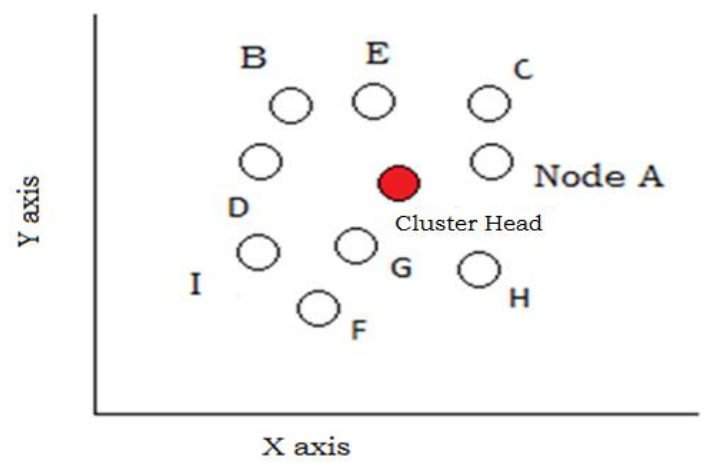

Fig.2. An example of clustering of nodes in EEDBLEACH

Find out of every nodes characteristic; Let an example compute sensor node D characteristic as shown in Figure .2. Let assume that attribute of each node such as Node $\mathrm{A}=\left(\mathbf{x}_{2}, \mathbf{y}_{2}\right)$, Node $\mathrm{B}=\left(\mathbf{x}_{3}, \mathbf{y}_{3}\right)$ and base station $/$ sink node $=\left(\mathbf{x}_{1}, \mathbf{y}_{1}\right)$.

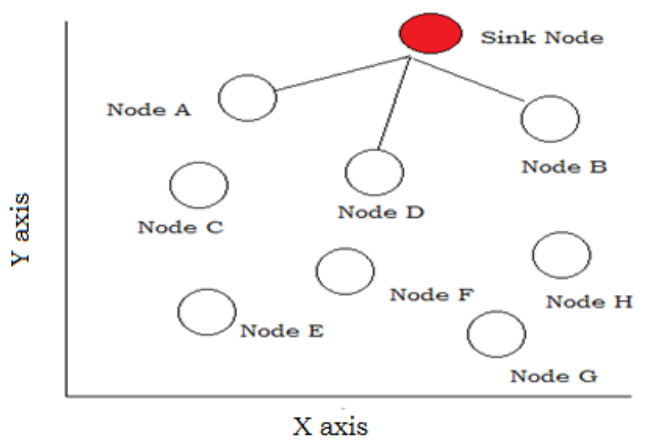

Fig.3. Indicate of nodes coordinates

To get attributes of the sensor hub D with good ways from hubs, for example, Node A, Node B and base station are sure measure of $a, b, c$. 
Let accept that hub $\mathrm{D}$ trademark is $\mathrm{D}=(\mathrm{x} 4, \mathrm{y} 4)$. As per the recipe computes good ways from hub $\mathrm{D}$ to sink hub is given condition

$$
1.13 \text {. }
$$$$
|\mathbf{D}-\operatorname{Sink}|^{2}=\left(\mathbf{x}_{4}-\mathbf{x}_{1}\right)^{2}+\left(\mathbf{y}_{4}-\mathbf{y}_{1}\right)^{2}=\mathbf{a}^{2}(1.13)
$$

Similarly below equations 2 and 3

$\left(\mathbf{x}_{4}-\mathbf{x}_{2}\right)^{2}+\left(\mathbf{y}_{4}-\mathbf{y}_{2}\right)^{2}=\mathbf{b}^{2}(1.14)$

$\left(x_{4}-x_{3}\right)^{2}+\left(y_{4}-y_{3}\right)^{2}=c^{2}$

To obtain equation subtracted equation 1.14 from equation 1.15 the $x_{4}$ equal to equation 1.16

$$
\mathrm{x}_{4}=\frac{\mathrm{a}^{2}-\mathrm{b}^{2}-\mathrm{x}_{12}+\mathrm{x}_{22}-\mathrm{y}_{12}+\mathrm{y}_{22}-2 \mathrm{y}_{4}\left(\mathrm{y}_{2}-\mathrm{y}_{1}\right)}{2\left(\mathrm{x}_{1}-\mathrm{x}_{2}\right)}
$$

Replacing with amount of $x_{-} 4$ in condition 1 , in the wake of setting coefficients of y_4 equivalent power, comprehend between two points which as stamped. These are supplanted with in condition 1, these figuring's have been accomplished for all sensor hubs in the network, base station and every sensor hub of qualities by utilizing these hubs trademark ought to decide separation of every hub from each other hubs. Staying other hub trademark additionally spared to comparative table, and afterward, contrasted and hubs in network region and the sensors hubs are isolated into equivalent region (bunch) in a network. This is structure as groups. The group sensor hub is in explicit condition. These sensor hubs might be in vertical line, level line or the two of them. Let expect that they are three conditions: first condition if hub is coming towards even line, accept that it is a base square part. Second condition if hub is coming towards vertical line, expect that it is left square part. At long last if hub coming towards both level and vertical lines, accept that it is base square part. In like manner all sensors hubs in the network will be an individual from one square region, expect that each square zone ought to be a group, in each bunch ought to be select group head.

To decision of group head will get the likelihood of bunch head dependent on residual vitality and normal separation of every hub in the network. To acquire normal separation of every hub from one another hubs in same bunch and put normal separation into the condition.

1.16 ,

Probability of cluster head $P_{n i}=\frac{E_{n i}}{m_{n i}}$

Where average distancem ${ }_{n i}=\frac{\sum_{j-1}^{n} d_{j}}{n}$

$\mathrm{n}$ is Number of sensor nodes in cluster $\mathrm{ni}=\mathrm{i}^{\text {th }}$ Node

$\mathrm{m}_{\mathrm{ni}}=$ distance with other nodes

$E_{n i}$ is remaining energy of each sensor node

Note that each hub separation with itself separation is 0 , from given conditions select few of the sensor hubs as bunch heads, and after that group head will apportion timespan to every part hub, so that inside its timeframe sensor hub output send detected message. Subsequent to getting information from individuals hubs the bunch heads gathered message send to base station/sink hub. In the wake of closure of time select as bunch head $(\mathrm{CH})$ for groups.

\section{EEDBLEACH protocol Steps}

- $\quad$ Nodes randomly deployed over a sensor field area

- Clusters are formed based on the predefined criteria

- Compute separation among hubs and furthermore

Base Station
- $\quad$ Determine the rest of the vitality of the hub

- $\quad$ Assign the best hub with reference to separate and remaining vitality of the hub to set $\mathrm{G}$.

- Nodes weighted choice likelihood and limit is determined.

- Each hub creates an arbitrary number and is contrasted and limit esteem. In the event that the produced worth is not as much as limit, then that hub will progresses toward becoming $\mathrm{CH}$

- If limit is under weighted determination likelihood then the hub is chosen as group head

- $\quad$ Cluster head offers notice to part hub as a $\mathrm{CH}$

- Cluster head get information from individuals

- Cluster head total

- data

- Cluster head transmit aggregated data to Base Station

- If energy of the selected cluster head becomes less than the threshold energy, then normal node will be considered for selection of cluster head.

- Same procedure is continued for next rounds

\section{RESULTS AND DISCUSSIONS}

The reenactment aftereffects of EEDBLEACH and LEACH protocols have been done by utilizing network test system (NS 2.35). The reenactment results were acquired by changing No. of hubs. The accompanying reenactment situation table 3.1 utilized for getting results.

Table: 1 Simulation Scenario

\begin{tabular}{|c|c|}
\hline Scenario parameter & Value \\
\hline Number of Nodes & $50,60,70,80,90,100$ \\
\hline Simulation area & $1000 \times 1000$ sq. M \\
\hline Routing Protocols & EEDBLEACH, LEACH \\
\hline Popt & 0.5 \\
\hline Traffic class & CBR \\
\hline Data Rate & $0.05 \mathrm{Mbps}$ \\
\hline Simulation time & 100 sec \\
\hline EO & $0.5 \mathrm{~J}$ \\
\hline Antenna & Omni Directional \\
\hline MAC & IEEE 802.11 \\
\hline
\end{tabular}

\section{a. Simulation results by varying number of nodes}

Figure shows the outcomes for execution measurements, for example, leftover vitality, vitality utilization, start to finish postponement, throughput and bundle conveyance proportion. The outcomes are acquired by fluctuating number of hubs 


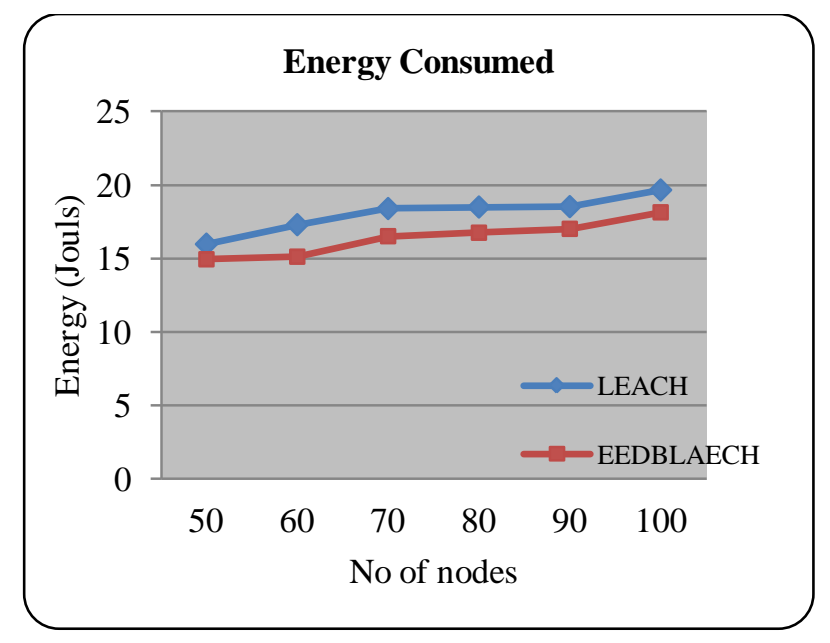

Fig 4. Energy Consumption Vs Number of nodes

Fig.4 shows that vitality utilization of EEDBLEACH and LEACH protocols when number of hubs are differed. The EEDBLEACH is less vitality utilization than LEACH convention. The bunch heads $(\mathrm{CH})$ are evenly chosen dependent on outstanding vitality and separation among hubs and sink hub, the EEDBLEACH convention has more vitality productive.

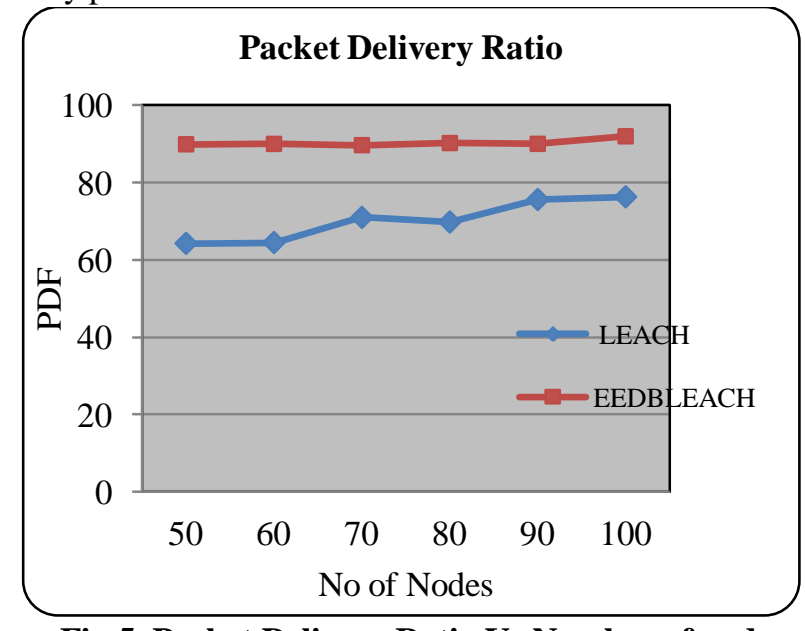

Fig.5. Packet Delivery Ratio Vs Number of nodes

The EEDBLEACH convention has higher bundle conveyance proportion when contrasted and LEACH protocols as appeared in above figure 5.The PDR is somewhat diminished with expanding number of hubs.

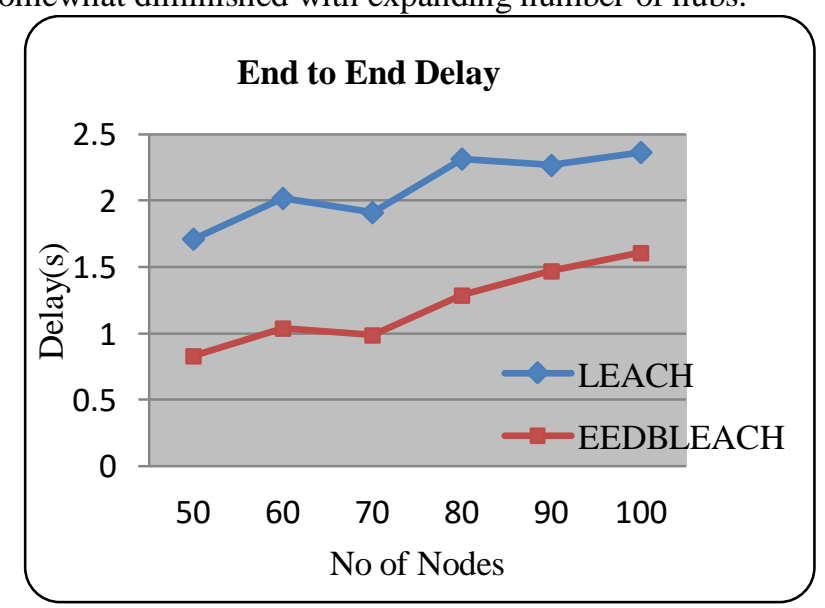

Fig.6. End to End Delay Vs No Nodes
Figure shows that end delay is increasing with increasing nodes. Obtained end to end delay for EEDBLAECH, LAECH protocols by varying No. of nodes i.e. from 50 to 100 nodes and node speed constant. The EEDBLAECH protocol is has less delay when compare with LAECH protocols as shown in figure 6 .

\section{CONCLUSIONS}

The fundamental objective of this exploration is to lessen the vitality utilization in wireless sensor networks utilizing vitality productive routing protocols. The recreation of proposed EEDBLEACH protocols is done utilizing network test system (ns 2. 35). The EEDBLEACH convention given better execution when contrast and LEACH convention. The vitality utilization diminished by $10 \%$ than $\mathrm{LEACH}$ convention, the parcel conveyance proportion likewise expanded by $8 \%$. The EEDBLEACH Protocol gives better execution understudies of vitality utilization. The Performance of Energy Efficient and Fault Tolerant Clustering Protocol.

\section{REFERENCES}

1. Can Tunca, Sinan Isik, Mehmet Yunus Donmez, and Cem Ersoy, "Ring Routing: An Energy-Efficient Routing Protocol for Wireless Sensor Networks with a Mobile Sink", IEEE transactions on mobile computing, vol. 14, issue no. 9, pp 1947-1960, september 2015.

2. .Akinsolu Ojuetimi Ifedayo, Mqhele Dlodlo "Variable Transmission Power Control in Wireless Ad-hoc Networks" IEEE 2015.

3. K. Vijayan and Arun Raaza "A Novel Cluster Arrangement Energy Efficient Routing Protocol for Wireless Sensor Networks",Indian Journal of Science and Technology, Vol 9(2),Vol. 9 issue. 2. pp 1-9, January 2016.

4. Monika Raghatate1 and Prof. Dipak W. Wajgi2, "AN ENERGY SAVING ALGORITHM TO PROLONG THE LIFETIME OF WIRELESS SENSOR NETWORK", International Journal of Wireless \& Mobile Networks (IJWMN) Vol. 6, No. 5,pp 32-44, October 2014

5. Mohammed Abo-Zahhad, Sabah M. Ahmed, Nabil Sabor and Shigenobu Sasaki, "A New Energy-Efficient Adaptive Clustering Protocol Based on Genetic Algorithm for Improving the Lifetime and the Life timeof Wireless Sensor Networks", International Journal of Energy, Information and Communications Vol.5, Issue 3, pp.47-72,2014

6. Omar N, Al Khayat, Siddeeq Y Ameen, Mohammed N Abdallah, "WSN Power Consumption using clustering and multiple access techniques", International Journal of Computer Applications, vol-87, No-9, Feb 2014.

7. Seyyed Aboalfazl Sayyadi, Ramin Senemar,Hossein Teimuri, "Elect Distance Efficient Cluster Heads for Improvement Performance of LEACH Protocol", 2nd International Conference on Computational Techniques and Artificial Intelligence (ICCTAI'2013), March 17-18, Dubai (UAE) 2013.

8. Soamsiri, "Multi-channel utilization algorithms for IEEE 802.15.4 based wireless network A survey", Engineering Journal, Vol-17, Issue3, July 2013. 
9. Kieu-Ha Phung, Bart Lemmens, Mihail Mihaylov, Lan Tran, Kris Steenhaut, "Adaptive Learning based scheduling in multichannel protocol for energy-efficient data-gathering wireless sensor networks", International Journal of Distributed Sensor Networks,.vol. 9 no. 2,pp111,February 2013.

10. O. Rehman, N. Javaid, B. Manzoor, A. Hafeez, A. Iqbal, M. Ishfaq "Energy Consumption Rate based Stable Election Protocol (ECRSEP) for WSNs" International Workshop on Body Area Sensor Networks (BASNet2013), Procedia Computer Science, pp 932 - 937, Elsevier ,2013

11. Surender et al., "Energy Efficient (EECP) Clustered Protocol for Heterogeneous Wireless Sensor Network" , International Journal of Advanced Research in Computer Science and Software Engineering vol. 3, issue 7, pp. 1448-1453, July - 2013.

12. Jin Wang, Zhongqi Zhang, Feng Xia, Weiwei Yuan and Sungyoung Lee," An Energy Efficient Stable Election-Based Routing Algorithm for Wireless Sensor Network", Vol. 13, pp 14301-14320; doi:10.3390/s131114301, Sensors 2013.

13. C.Divya, N.Krishnan , T.Gandhi mathy,"Energy Efficient Stable Election Protocol for Clustered Heterogeneous Wireless Sensor Networks", IOSR Journal of Computer Engineering (IOSR-JCE) Vol. 12, Issue 5 pp. 55-61, 2013.

14. Giuseppe Anastasi, Marco Conti, and Mario Di Francesco, "Extending the Lifetime of Wireless Sensor Networks Through Adaptive Sleep", IEEE Transactions on Industrial Informatics, vol. 5, no. 3, pp. 351-365, 2009.

15. Jin Wang, Zhongqi Zhang, Feng Xia, Weiwei Yuan and Sungyoung Lee, "An Energy Efficient Stable Election-Based Routing Algorithm for Wireless Sensor Networks", vol.13, pp. 14301-14320; doi:10.3390/s131114301, Sensors 2013.

16. Malay K Pakhira, "Finding number of clusters before finding clusters", Procedia Technology 4 27-37, published by Elsevier Ltd. 2012

17. Nazia Majadi, "U-LEACH: A Routing Protocol for Prolonging Lifetime of Wireless Sensor Networks", International Journal of Engineering Research and Applications, Vol. 2, Issue4, July-, pp.1649-1652, August 2012. 